\title{
Validation of Combustion Models for Lifted Hydrogen Flame
}

\author{
Ali Cemal Benim*, and Björn Pfeiffelmann \\ Center of Flow Simulation (CFS), Department of Mechanical and Process Engineering, Düsseldorf University of Applied Sciences, \\ Münsterstr. 156, D-40476 Düsseldorf, Germany
}

\begin{abstract}
Within a Reynolds Averaged Numerical Simulation (RANS) approach for turbulence modelling, a computational investigation of a turbulent lifted $\mathrm{H}_{2} / \mathrm{N}_{2}$ flame is presented. Various turbulent combustion models are considered including the Eddy Dissipation Model (EDM), the Eddy Dissipation Concept (EDC), and the composition Probability Density Function transport model (PDF) in combination with different detailed and global reaction mechanisms. Turbulence is modelled using the Standard k- $\varepsilon$ model, which has proven to offer a good accuracy, based on a preceding validation study for an isothermal $\mathrm{H}_{2} / \mathrm{N}_{2}$ jet. Results are compared with the published measurements for a lifted $\mathrm{H}_{2} / \mathrm{N}_{2}$ flame, and the relative performance of the turbulent combustion models are assessed. It is observed that the prediction quality can vary largely depending on the reaction mechanism and the turbulent combustion model. The best and quite satisfactory agreement with experiments is provided by two detailed reaction mechanisms applied with a PDF model.
\end{abstract}

\section{Introduction}

Power generation by gas and steam turbines [1] depend largely on the combustion process. Parallel to the efforts for exploiting new energy sources [2] as well as recovery techniques [3], combustion will continue to play an important role in power generation. This is true also for renewables, as biomass [4] plays an important role.

Combustion of hydrogen and hydrogen containing fuels occupies an important role in clean and efficient energy supply, environment protection and resource efficiency. Hydrogen offers an attractive alternative for storing excess energy in power generation from photovoltaics and wind energy. Furthermore, instead of combustion [5] the gasification of waste, biomass and coal [6] offers good possibilities for efficient and clean power generation. The so-called synthesis gas (syngas), which results as the product of gasification, contains, in addition to carbon monoxide and small fractions of methane, rather significant amounts of hydrogen. Additionally, there is a growing interest in nuclear energy based hydrogen production, i.e., using the nuclear power for electrolysis, thermochemical cycles or hybrid approaches to produce hydrogen [7]. From the environmental perspective, its subsequent combustion is most welcome since it produces no carbon dioxide.

Utilization of hydrogen or hydrogen blend fuels in combustion systems represents a great challenge. Hydrogen is extremely reactive and, compared to other gases, has different material properties, so that it can alter the combustion properties of the gas mixture even in small proportions. In premixed combustion, a potential problem is increased flashback propensity [8]. The counterpart of flashback is blow-off [9]. The forerunner of blow-off is the lift-off, as the flame root leaves the rim. Following the lift-off, with a further increase of the jet speed, a stabilized flame at a distance from the rim, i.e. a lifted flame can be obtained [10].

Computational analysis of turbulent lifted flames is a very challenging task, due to the modelling of turbulence and its interaction with chemistry [11]. For turbulence modelling, although the Large Eddy Simulation (LES) approach [12-14] is being increasingly used in practical applications, its adequate use in industrial development processes is still very challenging and the RANS approach [15] is still frequently preferred to this purpose. Given this, the present work is focused on the RANS methodology, and in the following review, RANS based approaches will be considered only.

Prediction of lifted turbulent jet flames is a demanding task. A partially premixed state is reached at the flame base, which leads to complex stabilization mechanisms [16]. In configurations, where the fuel jet is issuing into a hot coflow, like the presently investigated one, autoignition emerges as a further possible mechanism of stabilization. Thus, the applied turbulent combustion model should sufficiently accommodate for the mentioned effects.

As the turbulent combustion model, Cabra et al. [17] applied a composition Probability Density Function transport model [18] (PDF) in predicting a lifted methane flame. For lifted hydrogen flames, the MIL (Modèle Intermittent Lagrangien) [19], the unsteady flamelet / progress variable [20], and the Conditional Moment Closure (CMC) [21] models were applied [22]. Several researchers used PDF as well as Eddy Dissipation Concept (EDC) [23] approaches.

\footnotetext{
* Corresponding author: alicemal@prof-benim.com
} 
As outlined above, different and quite sophisticated models have already been used to predict turbulent, lifted hydrogen flames.

However, to the authors' opinion, there is still need for further investigation. The purpose of the present paper is to present a "coherent" validation study, for a cascade of different turbulent combustion modelling strategies in a wide range. This coherent validation study based on the consistent/comparable strategies in all further aspects of the mathematical modelling, numerical methods and gridding is believed to be of additional value to the research community, as it will provide a basis for a direct comparison of a wide range of turbulent combustion models, unmasked by the other effects, for the present problem.

\section{Problem definition}

The "main" considered test case is the atmospheric, lifted flame of a turbulent $\mathrm{H}_{2} / \mathrm{N}_{2}$ jet in vitiated coflow, which was experimentally investigated by Cabra et al. [24]. This comprises a free jet of $\mathrm{H}_{2} / \mathrm{N}_{2}$ mixture (with $\mathrm{H}_{2}$ volume fraction of $25.37 \%$ ) in a co-flow of exhaust gases stemming from lean hydrogen combustion (with oxygen volume fraction of $14.74 \%$ ).

In comparing the predictions with the experimental results, mainly, the lift-off heights of the flames for different values of the coflow temperatures are monitored. For this purpose, the experimental data of $\mathrm{Wu}$ et al. [25] is considered which was obtained on an experimental setup, which was equivalent to that of Cabra et al [24].

For selecting the turbulence model to be used, a study has been carried out, where turbulence models are validated in an isolated manner from the combustion model, on an isothermal, non-reacting test case, which, however, resembles the setup of the main, combusting test case, in so far that the gas composition (containing $\mathrm{H}_{2}$ ), and, thus, density, is variable.

As test case for this purpose, the measurements of Sautet and Stepowski [26] are considered. The test rig was an open, atmospheric one at ambient conditions. In the experiments, non-reacting turbulent jets of $\mathrm{H}_{2} / \mathrm{N}_{2}$ mixtures discharging into a coflow air stream were investigated.

\section{Models}

The general-purpose, finite volume based CFD code ANSYS Fluent 18.0 [27] is used in the computational analysis of the problem.

\subsection{Outline}

A two-dimensional, axisymmetric formulation is used. The medium is considered to be an ideal gas mixture with Newtonian behavior. Buoyancy effects are neglected, which is reasonable due to the prevailing rather high Froude numbers [28]. The radiative heat transfer [29] is also neglected and the reacting system is assumed to be adiabatic. An accurate modelling of the molecular material properties is attempted. For all species, the specific heat capacities are represented by a pair of (low and high temperature ranges) fourth order polynomials of temperature [30]. The viscosities, thermal conductivities, as well as the multi-component diffusion coefficients are calculated according to the kinetic theory [9].

The flow turbulence is described within a RANS framework [15], as already mentioned above. Among the two-equation turbulence models the specific dissipation rate (omega, $\omega$ ) based models have gained popularity $[15,31]$. However, since the present problem is of completely free-shear type, among the two-equation models, the dissipation rate (epsilon, $\varepsilon$ ) based models are considered only, in particular, the Standard k- $\varepsilon$ [27,32], the RNG k- $\varepsilon$ [27,33], and the Realizable k- $\varepsilon$ models $[27,33,34]$. For scalar turbulent fluxes, the gradientdiffusion approximation is used assuming constant turbulent Schmidt numbers. For the latter, 0.85 is used for the energy equation, whereas 0.7 is assumed for the further scalars.

The velocity-pressure coupling is treated by the SIMPLEC scheme [27]. For the discretization of the convective terms, the QUICK scheme [27] is used, which is considered to possess formal accuracy of third order. As no under-relaxation is applied to pressure, the under-relaxation factors range between 0.4-0.7 and 0.81.0 for the velocities and the scalar quantities, respectively.

For convergence, the threshold value for the normalized residual has been set to $10^{-8}$ for the energy equation and to $10^{-5}$ for the remaining equations.

\subsection{Combustion models}

As single-step global mechanisms, those of Kudriakov et al. [36] (KU) and Marinov et al. [37] (M) are considered (comprising the main species, $\mathrm{H}_{2}, \mathrm{O}_{2}, \mathrm{H}_{2} \mathrm{O}$ ), where the former and latter consider an irreversible and a reversible reaction, respectively.

As detailed reaction mechanisms, four mechanisms are considered, each of which comprising a large number of elementary reactions (approx. 20 without counting the reverse reactions) between eight species, names, $\mathrm{H}_{2}, \mathrm{O}_{2}$, $\mathrm{H}_{2} \mathrm{O}, \mathrm{H}, \mathrm{O}, \mathrm{OH}, \mathrm{HO}_{2}, \mathrm{H}_{2} \mathrm{O}_{2}$. The considered detailed mechanisms are the Gri-Mech 3.0 [38] (GRI), the mechanisms of Li et al. [39] (LI), Conaire et al. [40] $(\mathrm{CON})$ and Keromnes et al. [41] (KER).

For purely mixing controlled combustion, the timeaveraged consumption rate is calculated, in Eddy Dissipation Model (EDM), from the dissipation rate of turbulence eddies [42]. The chemical kinetics effects $(\mathrm{K})$ are taken in an ad-hoc manner into account, calculating the rate from an Arrhenius expression neglecting fluctuations [9], comparing the two rates and taking the smaller one [27].

As an improved version of the eddy dissipation idea is the Eddy Dissipation Concept (EDC), where the timeaveraged conversion rate is calculated by taking the mixing and kinetics effects in a combined manner into 
account, in a more sophisticated way, treating the small turbulent scales to behave as well stirred reactors $[27,43]$.

In the composition PDF transport (PDF) model, for obtaining the averaged values thermochemical variables a single-point, joint probability density function is obtained from its transport equation, which is derived from the governing equations under the application of some closure models [18,27].

\section{Results}

\subsection{Isothermal turbulent flow}

The solution domain is two-dimensional axisymmetric, having rectangular shape in plane of the axial $(\mathrm{x})$ and radial ( $\mathrm{r}$ ) coordinates.

The domain is starting at the exit plane of the jets, extending $22 \mathrm{~d}$ in the axial direction. Its radial extension is $11 \mathrm{~d}$. The inlet boundaries representing the central and coaxial jet are placed on the left boundary of the domain $(\mathrm{x}=0)$, whereas the right boundary $(\mathrm{x}=22 \mathrm{~d})$ is defined to be pressure boundary with a prescribed constant pressure and zero gradient conditions for the remaining variables. The lower $(\mathrm{r}=0)$ boundary is a symmetry axis, whereas the upper one ( $\mathrm{r}=11 \mathrm{~d})$ is also defined to be a symmetry surface.

On the left boundary $(x=0)$, the part that surrounds the annular jet is defined to be a pressure boundary, again, (ambient pressure) that allows an inflow, i.e. the suction of ambient air by the ejector effect. At inlet, the measured values are prescribed as the inlet boundary conditions. Boundary conditions of turbulence quantities are derived from assumed turbulence intensities and length scales.

Computational grids are generated as structured, rectangular grids, with axial and radial concentration of the nodes near the jet inlet. For determining the adequate grid resolution, a grid independence study is performed. In the grid independence study, the Standard k- $\varepsilon$ model is used as the turbulence model.

Table 1 displays the variation of the potential core length (L) with grid fineness, where $\mathrm{N}$ is the total number of nodes. One can see that sufficient grid independence is achieved for $\mathrm{N} \geq 5,000$. In the further calculations for the validation of turbulence models, the finest grid is used, which had 16,200 nodes.

The predicted variations of the half value radius $(\delta)$ at the axial position of $\mathrm{x} / \mathrm{d}=20$ are compared with the experimental values in Table 2.

One can see that the predictions delivered by the Standard k-E model agree rather well with the experiments, better than the Realizable and $\mathrm{RNG}$ versions, for the present, variable density $\mathrm{H}_{2} / \mathrm{N}_{2} /$ Air jet (Table 2). Thus, the Standard k- $\varepsilon$ model is selected.

Table 1. Potential core length as function of total number of grid nodes.

\begin{tabular}{cccccccc}
\hline $\mathrm{N}$ & 1375 & 2450 & 2925 & 3250 & 4590 & 6405 & 16200 \\
\hline $\mathrm{L} / \mathrm{d}$ & 2.08 & 2.64 & 2.79 & 3.06 & 3.50 & 3.51 & 3.51 \\
\hline
\end{tabular}

Table 2. Variation of half-value radius $(\delta)$ along jet axis.

\begin{tabular}{ccc}
\cline { 2 - 3 } & $\delta / \mathrm{d}$ & $\%$ Error \\
\hline Experiment & 1.36 & - \\
Standard k- $\varepsilon$ & 1.45 & $6.6 \%$ \\
Realizable k- $\varepsilon$ & 1.55 & $13.9 \%$ \\
RNG k- $\varepsilon$ & 1.62 & $19.1 \%$ \\
\hline
\end{tabular}

\subsection{Flame}

Similar to the isothermal test case, the solution domain consists of a cylinder, the bottom of which is placed at the jet exit. The axial coordinate (x) extends along the axis, in the main flow direction, with $\mathrm{x}=0$ placed at the jet exit (jet inlet boundary is centered at the cylinder bottom). The domain size in the radial and axial directions are about $20 \mathrm{~d}$ and $80 \mathrm{~d}$, respectively. The cylinder bottom is covered by two inlet boundaries, i.e. a central (jet), and an annular (coflow) one. Both inlets are separated by a thin, ring shaped wall boundary, representing the nozzle the lip. The top of the cylinder is defined as the outlet boundary, whereas the jacket of the cylinder is assumed to be an impermeable slip boundary. At the outlet boundary, a constant static pressure is prescribed, along with vanishing normal-gradient conditions for the remaining quantities. At the inlets, top-hat profiles are prescribed for all convectivediffusively transported variables, in accordance with the measured values. For turbulence quantities, a turbulence intensity of $4 \%$ is assumed at the both inlets. The jet diameter and the size of the individual holes in the outer disk are taken as basis in assuming the length scale for the jet, and coflow, respectively.

Computational grids are generated as structured, rectangular grids, with axial and radial concentration of the nodes near the inlets as well as in the central and mixing zones. The grid independence study is performed using the $\mathrm{EDM}+\mathrm{K}$ turbulent combustion model in combination with the global reaction mechanism (KU).

Table 3 presents the variation of the predicted centerline temperature $(\mathrm{T})$ at ten diameters downstream the jet inlet $(\mathrm{x} / \mathrm{d}=10)$ for six different grids with changing number of total nodes $(\mathrm{N})$. One can see that sufficient grid independence is achieved for the finer grids. In the further calculations, the finest grid having 16,000 cells is used.

The temperature and oxygen mole fraction fields predicted by the $\mathrm{EDM}+\mathrm{K}$ model, using the global mechanism $M$ are presented in Figure 1 for the coflow temperature $\left(\mathrm{T}_{\mathrm{CO}}\right)$ of $1060 \mathrm{~K}$.

The lifted flame can easily be recognized in the temperature field (Fig. 1a). In Fig. 1b, one can see that oxygen penetrates into the fuel jet along the lift-off distance, and, is, then, rapidly consumed by the combustion reactions starting at the flame root, causing a local oxygen depleted zone.

Table 3. Centerline temperature at $\mathrm{x} / \mathrm{d}=10$ as function of total number of grid nodes.

\begin{tabular}{ccccccc}
\hline $\mathrm{N}$ & 2436 & 4260 & 7525 & 9900 & 12480 & 16000 \\
\hline $\mathrm{T}(\mathrm{K})$ & 573 & 456 & 410 & 392 & 384 & 384 \\
\hline
\end{tabular}




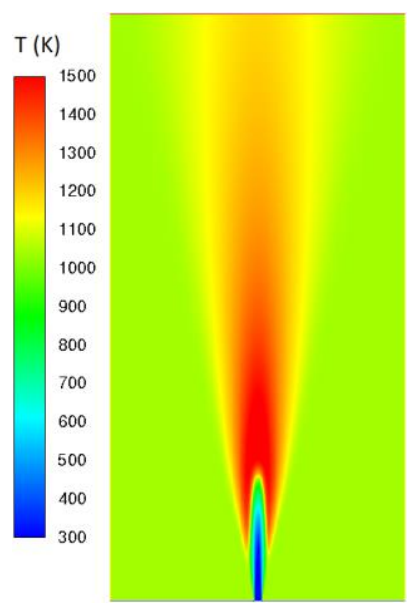

(a)
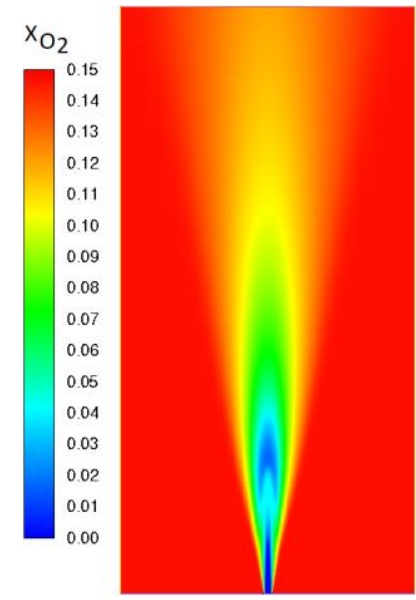

(b)
Figure 1. Predicted fields of (a) temperature and (b) oxygen mole fraction by $\mathrm{EDM}+\mathrm{K}-\mathrm{M}$ for $\mathrm{T}_{\mathrm{CO}}=1060 \mathrm{~K}$.

Inspecting the data of $\mathrm{Wu}$ et al. [6] one can deduce that the measured lift-off heights in their dependence to the temperature of the coflow-stream can be represented by the following relationship with a quite good accuracy

$$
\frac{\mathrm{h}}{\mathrm{d}}=81.37\left(\frac{\mathrm{T}_{\mathrm{CO}}}{1000}\right)^{-68.12}
$$

with a coefficient of determination of 0.98 .

Predicted lift-off heights by the single-step reaction mechanism (M), in combination with EDM, EDC models, i.e. by EDM+K-M, and EDC-M, as nondimensionalized by the central jet diameter, and the experimental results (EXP), as represented by the correlation expressed by Eq. (1), are presented in Table 4 , for different coflow temperatures.

One can see that the calculations predict qualitatively the right trend, i.e. decreasing lift-off height with increasing temperature. However, this trend is strongly underpredicted by the calculations and the quantitative deviations from the measurements are quite large.

Similarly, the calculated lift-off heights by detailed reaction mechanisms (CON, LI, KER), in combination with EDC, i.e. EDC-CON, EDC-LI and EDC-KER models are compared with experiments in Table 5. Empty boxes in the table indicate that no flame could be predicted for the corresponding temperature. One can see that the detailed mechanisms with EDC predict too small lift-off heights at high temperatures, with a very sudden and rapid increase with decreasing temperature followed by blow-off, beyond a certain value. Using the reaction

Table 4. Lift-of heights predicted by EDM+K-M, EDC-M, compared with experiments.

\begin{tabular}{cccc}
\cline { 2 - 4 } & \multicolumn{3}{c}{$\mathrm{h} / \mathrm{d}$} \\
\hline $\mathrm{T}_{\mathrm{CO}}(\mathrm{K})$ & $\mathrm{EXP}$ & $\mathrm{EDM}+\mathrm{K}-\mathrm{M}$ & $\mathrm{EDC}-\mathrm{M}$ \\
\hline 1010 & 41.3 & 7.5 & 20.4 \\
1020 & 21.1 & 6.5 & 15.9 \\
1030 & 10.9 & 5.2 & 11.4 \\
1040 & 5.6 & 4.1 & 7.5 \\
1045 & 4.1 & 3.7 & 6.3 \\
\hline
\end{tabular}

Table 5. Lift-off heights predicted by EDM-CON, EDC-LI, EDC-KER, compared with experiments.

\begin{tabular}{ccccc}
\cline { 2 - 5 } & \multicolumn{4}{c}{$\mathrm{h} / \mathrm{d}$} \\
\hline $\mathrm{T}_{\mathrm{CO}}(\mathrm{K})$ & EXP & EDC-CON & EDC-LI & EDC-KER \\
\hline 1010 & 41.3 & - & - & - \\
1020 & 21.1 & - & 24.8 & 21.4 \\
1030 & 10.9 & 38.9 & 3.0 & 2.8 \\
1040 & 5.6 & 3.8 & 1.0 & 0.8 \\
1045 & 4.1 & 2.0 & 0.7 & 0.6 \\
\hline
\end{tabular}

mechanism GRI with EDC, no flame could be predicted for the listed temperatures.

Calculated lift-off heights by detailed reaction mechanisms (GRI, CON, LI, KER), in combination with PDF, i.e. PDF-GRI, PDF-CON, PDF-LI and PDF-KER models are compared with experiments in Table 6 . Empty boxes in the table indicate that no flame could be predicted for the corresponding temperature. With the GRI mechanism, a flame could be predicted only for the highest temperatures, however, with an extremely large lift-off height. With the mechanism CON, a flame is predicted for all temperatures, with an overprediction of the lift-off height throughout. The mechanisms LI and KER show a very good agreement with each other (except for the lowest temperature $1010 \mathrm{~K}$, where PDFKER overpredicts PDF-LI) and a quite fair agreement with the measurements. For high temperatures, PDF-LI and PDF-KER predict very close values to the experiments, and the degree of agreement is decreases as the temperature is reduced and the lift-off height increases. Still the overall agreement of PDF-LI and PDF-KER with the experiments is much better than that of the other simulation methods considered here.

\section{Conclusions}

A computational investigation of a turbulent lifted $\mathrm{H}_{2} / \mathrm{N}_{2}$ flame is presented, based on a RANS turbulence modelling approach, using the Standard k- $\varepsilon$ model. Detailed reaction mechanisms of $\mathrm{Li}$ et al. [39] and Keromnes et al. [41] applied with PDF are observed to deliver the best predictions of the lift-off height (h) as function of the coflow temperature $\left(\mathrm{T}_{\mathrm{CO}}\right)$. The mechanism of Conaire et al. [40] with PDF overpredicts $\mathrm{h}$. These reaction mechanisms do not perform that well, when applied with EDC, underpredicting $h$ for high $\mathrm{T}_{\mathrm{CO}}$ with an abrupt increase of $h$ for $T_{C O}$ lower than a certain value, followed by a too early blow-off. The GRI Mech 3.0 [38] predicted a blow-off for all $\mathrm{T}_{\mathrm{CO}}$ when applied with EDC, and a lifted flame, with an extremely

Table 6. Lift-off heights predicted by PDF-GRI, PDF-CON, PDF-LI, PDF-KER, compared with experiments.

\begin{tabular}{cccccc} 
& \multicolumn{5}{c}{$\mathrm{h} / \mathrm{d}$} \\
\hline $\mathrm{T}_{\mathrm{CO}}(\mathrm{K})$ & EXP & PDF-GRI & PDF-CON & PDF-LI & PDF-KER \\
\hline 1010 & 41.3 & - & 79.1 & 54.6 & 65.0 \\
1020 & 21.1 & - & 55.1 & 30.6 & 31.4 \\
1030 & 10.9 & - & 33.9 & 14.0 & 13.9 \\
1040 & 5.6 & 59.7 & 15.9 & 7.4 & 7.9 \\
1045 & 4.1 & 50.1 & 11.5 & 5.7 & 5.6 \\
\hline
\end{tabular}


overpredicted $\mathrm{h}$, for $\mathrm{T}_{\mathrm{CO}}>1045 \mathrm{~K}$, when applied with PDF. The global mechanism of Marinov et al. [37] applied with $\mathrm{EDM}+\mathrm{K}$ and $\mathrm{EDC}$ could deliver the trend of increasing $h$ with decreasing $\mathrm{T}_{\mathrm{CO}}$, whereas the EDC version delivered quantitatively better results. The global mechanism of Kudriakov et al. [41] with EDM+K predicted attached flames for all $\mathrm{T}_{\mathrm{CO}}$.

\section{References}

1. A.C. Benim, M. Geiger, S. Doehler, M. Schoenenberger, H. Roemer, "Modelling the flow in the exhaust hood of steam turbines under consideration of turbine-exhaust hood interaction", in: Proceed. 1st European Conferece on Turbomachinery - Fluid Dynamic and Thermodynamic Aspects: Computational Methods, Erlangen, Germany, March 1-3, 1995, Book Series: VDI Berichte, Vol. 1185, pp.343-357 (VDI Verlag, Duesseldorf, 1995)

2. E. Bellos, I. Danill, C. Tzivanidis, "A cylindrical insert for parabolic trough solar collector", International Journal of Numerical Methods for Heat \& Fluid Flow, 29(5), pp.1846-2876 (2019)

3. D.G. Ebling, A. Krumm, B. Pfeiffelmann, J. Gottschald, J. Bruchmann, A.C. Benim, M. Adam, R. Labs, R.R. Herbertz, A. Stunz, "Development of a system for thermoelectric heat recovery from stationary industrial processes", Journal of Electronic Materials, 45(7), pp. 3433-3439 (2016)

4. S. Cordiner, A. Manni, V. Mulone, V. Rocco, "Biomass furnace study via 2D numerical modeling", International J. Numerical Methods for Heat \& Fluid Flow, 26(2), pp. 515-533 (2016)

5. J.P. Kim, U. Schnell, G. Scheffknecht, A.C. Benim, "Numerical modelling of MILD combustion for coal", Progress in Computational Fluid Dynamics An International Journal, 7(6), pp.337-346 (2007).

6. S. Cordiner, A. Manni, V. Mulone, V. Rocco, "Biomass pyrolysis modeling of systems at laboratory scale with experimental validation", International Journal of Numerical Methods for Heat \& Fluid Flow, 28(2), pp. 413-438 (2018)

7. R. S. El-Emam, I. Khamis, "Advances in nuclear hydrogen production: Results from an IAEA international collaborative research project", International Journal of Hydrogen Energy, https:// doi.org/10.1016/j.ijhydene.2018.04.012 (2018)

8. A.C. Benim, K.J. Syed, Flashback Mechanisms in Lean Premixed Gas Turbine Combustion, (Academic Press, Cambridge, 2014).

9. S.R. Turns, An Introduction to Combustion, 3rd Ed. (McGrawHill, New York, 2012)

10. C. J. Lawn, "Lifted flames on fuel jets in co-flowing air", Progress in Energy and Combustion Science, 35, pp. 1-30 (2009)

11. P.A. Libby, F.A. Williams, Turbulent Reacting Flows (Academic Press, London, 1994)
12. P. Sagaut, Large Eddy Simulation for Incompressible Flows, 3rd Ed., (Springer, Berlin, 2006)

13. A.C. Benim, M.P. Escudier, A. Nahavandi, A.K. Nickson, K.J. Syed, F. Joos, "Experimental and numerical investigation of isothermal flow in an idealized swirl combustor", International Journal of Numerical Methods for Heat \& Fluid Flow, 20(3), pp.348-370 (2010)

14. A.C. Benim, S. Iqbal. W. Meier, F. Joos, A. Wiedermann, "Numerical investigation of turbulent swirling flames with validation in a gas turbine model combustor", Applied Thermal Engineering, 110, pp.202-212 (2017)

15. P.A. Durbin, B.A. P. Reif, Statistical Theory and Modelling for Turbulent Flows, 2nd Ed. (Wiley, Chichester, 2011)

16. R.W. Schefer, M. Namazian, J. Kelly, "Stabilization of lifted turbulent-jet flames", Combustion and Flame, 99, pp.75-86 (1994).

17. R. Cabra, J.Y. Chen, R.W. Dibble, A.N. Karpetis, R. S. Barlow, "Lifted methane-air jet flames in a vitiated coflow", Combustion and Flame, 143, pp.491-506 (2005).

18. S.B. Pope, "Computations of turbulent combustion: Progress and challenges", Proceedings of the Combustion Institute, 23, pp. 591-612 (1991)

19. M. Gonzalez, R.A. Borghi, "A Lagrangian intermittent model for turbulent combustion theoretical basis and comparisons with experiments", in: Durst, F., Launder B.E., Reynolds, W.C., Schmidt, F.W. and Whitelaw, J.H. (Eds.) Turbulent Shear Flows 7 (Springer, Berlin, 1991)

20. H. Pitsch, M. Ihme, "An unsteady flamelet/progress variable method for LES of nonpremixed turbulent combustion", AIAA Paper 2005-557 (2005)

21. A.Y. Klimenko, R.W. Bilger, "Conditional moment closure for turbulent combustion", Progress in Energy and Combustion Science, 25, pp.595-687 (1999)

22. S. De, A. De, A. Jaiswal, A. Dash, "Stabilization of lifted hydrogen jet diffusion flame in a vitiated coflow: Effects of jet and coflow velocities, coflow temperature and mixing", International Journal of Hydrogen Energy, 4(33), pp.15026-15042 (2016)

23. I.R. Gran, B.F. Magnussen,"A numerical study of a bluff-body stabilized diffusion flame. Part 2 . Influence of combustion modeling and finite-rate chemistry", Combustion Science and Technology, 119, pp.191-217 (1996)

24. R. Cabra, T. Myhrvold, J.Y. Chen, R.W. Dibble, A. N. Karpetis, R. S. Barlow, "Simultaneous laser Raman-Rayleigh-LIF measurements and numerical modelling results of a lifted turbulent $\mathrm{H}_{2} / \mathrm{N}_{2}$ jet flame in a vitiated coflow", Proceedings of the Combustion Institute, 29, pp. 1881-1888 (2002).

25. Z. Wu, S.H. Starner, R.W. Bilger, "Lift-off heights of turbulent $\mathrm{H}_{2} / \mathrm{N}_{2}$ jet flames in a vitiated coflow", Proc. 2003 Australian Symp. on Combustion and the 
$8^{\text {th }}$ Australian Flame Days, Monash University, Melbourne Australia, 8,9 December, 2003.

26. J.C. Sautet, D. Stepowski, "Dynamic behavior of variable density, turbulent jets in their near development field", Physics of Fluids, 7 (11) pp.2796-2806 (1995).

27. ANSYS $®$ FLUENT 18.0, www.ansys.com

28. R.M.C. So, H. Aksoy, "On vertical turbulent buoyant jets", International Journal of Heat and Mass Transfer, 36(13), pp.3187-3200 (1993).

29. A.C. Benim, "A finite element solution of radiative heat transfer in participating media utilizing the moment method", Computer Methods in Applied Mechanics and Engineering, 67(1), pp.1-14 (1988)

30. R.J. Kee, F.M. Rupley, J.A. Miller, "The Chemkin Thermodynamic Data Base", Sandia Report, SAND87-8215B (1991)

31. F.R. Menter, "Two-equation eddy-viscosity turbulence models for engineering applications", AIAA Journal, 32 (1994)

32. B.E. Launder, D.B. Spalding, "The numerical computation of turbulent flows", Computer Methods in Applied Mechanics and Engineering, 3, pp.269289 (1972)

33. V. Yakhot, S.A. Orszag, "Renormalization group analysis of turbulence", Journal of Scientific Computing, 1(1), pp.3-51 (1986)

34. T.J. Shih, W.W. Liou, A. Shabbir, Z. Yang, J. Zhu, 1995. "A new k- $\varepsilon$ model for high Reynolds number turbulent flows - Model development and validation", Computers and Fluids, 24(3), pp-227-238 (1995)

35. H. Chattopadhyay, A.C. Benim, "Turbulent heat transfer over a moving surface due to impinging slot jets", Journal of Heat Transfer - Transactions of the ASME, 133(10), Article Nr.: 104502, 5 pages, doi:10.1115/1.4004075 (2011)

36. S. Kudriakov, E. Studer, C. Bin, "Numerical simulation of the laminar hydrogen flame in the presence of a quenching mesh", International Journal of Hydrogen Energy, 36, pp.2555-2559 (2011)

37. N.M. Marinov, C.K. Westbrook, W.J. Pitz, "Detailed and Global Chemical Kinetics Model for Hydrogen" Report UCRL-JC-120677, Lawrence Livermore National Laboratory, California (1995)

38. http://combustion.berkeley.edu/gri-mech/

39. J.Li, Z.Zhao, A.Kazakov, F.L. Dryer, "An updated comprehensive kinetic model of hydrogen combustion", International Journal of Chemical Kinetics, 36, pp. 566-575 (2004)

40. M.O. Conaire, H.J. Curran, J.M. Simmie, W.J. Pitz, C. K. Westbrook, "A comprehensive modeling study of hydrogen oxidation, International Journal of Chemical Kinetics, 36, pp-603-622 (2004)

41. A. Keromnes, W.K. Metcalfe, K.A. Heufer, N. Donohoe, A.K. Das, C.J. Sung, J. Herzler, C. Naumann, P. Griebel, O. Mathieu, M.C. Krejci, E.L.
Petersen, W.J. Pitz, J.J. Curran, "An experimental and detailed chemical kinetic modeling study of hydrogen and syngas mixture oxidation at elevated pressures", Combustion and Flame, 160(6), pp.9951011 (2013)

42. B.F. Magnussen, B.H. Hjertager, "On mathe-matical models of turbulent combustion with special emphasis on soot formation and combustion", Proc. $16^{\text {th }}$ Symp. (Int.) Combustion (The Combustion Institute, Pittsburgh, PA, 1976) pp.719-729

43. I.R. Gran, B.F. Magnussen,"A numerical study of a bluff-body stabilized diffusion flame. Part 2 . Influence if combustion modeling and finite-rate chemistry", Combustion Science and Technology, 119, pp.191-217 (1996) 